\title{
TITLE:
}

\section{Analytic derivation of the map of null rays passing near a naked singularity}

\author{
$\operatorname{AUTHOR}(\mathrm{S}):$ \\ Tanaka, T; Singh, TP
}

\section{CITATION:}

Tanaka, T ...[et al]. Analytic derivation of the map of null rays passing near a naked singularity. PHYSICAL REVIEW D 2001, 63(12): 124021.

\section{ISSUE DATE:}

2001-06-15

URL:

http://hdl.handle.net/2433/49994

RIGHT:

Copyright 2001 American Physical Society 
PHYSICAL REVIEW D, VOLUME 63, 124021

\title{
Analytic derivation of the map of null rays passing near a naked singularity
}

\author{
Takahiro Tanaka \\ Yukawa Institute for Theoretical Physics, Kyoto University, Kyoto 606-8502, Japan \\ T. P. Singh \\ Yukawa Institute for Theoretical Physics, Kyoto University, Kyoto 606-8502, Japan \\ and Tata Institute of Fundamental Research, Homi Bhabha Road, Mumbai 400 005, India
}

(Received 7 November 2000; published 25 May 2001)

\begin{abstract}
Recently the energy emission from a naked singularity forming in spherical dust collapse has been investigated. This radiation is due to particle creation in a curved spacetime. In this discussion, the central role is played by the mapping formula between the incoming and the outgoing null coordinates. For the self-similar model, this mapping formula has been derived analytically. But for the model with $C^{\infty}$ density profile, the mapping formula has been obtained only numerically. In the present paper, we argue that the singular nature of the mapping is determined by the local geometry around the point at which the singularity is first formed. If this is the case, it would be natural to expect that the mapping formula can be derived analytically. In the present paper, we analytically rederive the same mapping formula for the model with $C^{\infty}$ density profile that has been earlier derived using a numerical technique.
\end{abstract}

DOI: 10.1103/PhysRevD.63.124021

PACS number(s): 04.20.Dw, 04.70.Dy

\section{INTRODUCTION}

There are many known examples in the literature showing that naked singularities can form from regular initial data, in the classical gravitational collapse of a bounded object. Quantum effects analogous to Hawking radiation are expected to play an important role in determining the final outcome of such a collapse. A study of such effects has received some attention in recent years. The most notable feature of these studies is that if a massless scalar field is quantized on the background of a star forming a naked singularity, the outgoing quantum flux of the scalar field can be calculated in the geometric optics approximation [1-5], and in $2 \mathrm{D}$ models [6-8]. This quantum flux can be shown to diverge on the Cauchy horizon. An interpretation of this divergence has recently been given by [9].

The calculation of the quantum flux in the geometric optics approximation crucially relies on the determination of the map between ingoing null rays coming in from $\mathcal{I}^{-}$and outgoing null rays going to $\mathcal{I}^{+}$. This map can be calculated in a straightforward manner for the model of self-similar spherical dust collapse. However, when the self-similarity condition is dropped, the calculation becomes difficult and the map has so far only been determined numerically.

In this paper we argue that this map is determined, not by the global geometry, but by the local geometry around the point at which the singularity is first formed. It is then possible to determine the map analytically.

In this paper, we solve the radial null rays in the TolmanBondi spacetime approximately. These null rays naturally define a map between the incoming and outgoing null coordinates, $u$ and $v$. As noted above, this map plays a crucial role in calculating the energy emission due to the quantum effect in curved spacetime in the geometrical optics approximation.

For the self-similar model with a stellar surface on which the background solution is matched to the Schwarzschild metric, this map was determined analytically [2]. It was shown that the map takes the form of a power law: $u_{0}-u$ $\propto\left(v_{0}-v\right)^{\gamma}$, where $u=u_{0}$ and $v=v_{0}$ are outgoing and incoming null rays passing through the point at which the singularity is first formed.

On the other hand, such a map was not derived analytically for the more generic so-called $\rho_{2}$ models for which we have non-vanishing second derivative of the density profile at the center before the formation of naked singularity. $\mathrm{Nu}-$ merically, the map was shown $[3,4]$ to be given as $d^{2}\left(u_{0}\right.$ $-u) / d v^{2} \propto\left(v_{0}-v\right)^{-1 / 2}$.

In this paper, we would like to show two things. First we shall point out that it is not necessary to study the global solution for radial null geodesics far from the singularity for the purpose of investigating the singular behavior of the map. We shall show that the basic feature of the map can be extracted just by considering a small region near the point at which the singularity is first formed.

To demonstrate this, we propose an alternative map. Let us consider sending radial incoming null rays from an observer on a comoving shell. These null rays are reflected at the center, and come back to the same comoving observer. A radial null geodesic crosses a comoving shell located at a fixed comoving radius $r$ twice before and after the reflection at the center. Thus these null rays define a map between the sending time and the receiving time measured by the proper time for the comoving observer. We shall show that this map possesses the same structure of singularity as the previously investigated map between null coordinates. In defining this map, we will see that the radius of the comoving shell can be chosen arbitrary small. One would expect such a result because there is no singular feature in the map between ticks on a comoving shell at a finite distance and that of the null coordinates naturally defined at infinity.

Secondly, using the same map, we show that the structure of singularity for $\rho_{2}$ models can be obtained analytically.

Throughout this paper, we use the units $8 \pi G=1$ and $c$ $=1$. 


\section{SELF-SIMILAR CASE}

The marginally bound Tolman-Bondi dust collapse is described by the metric

$$
d s^{2}=d t^{2}-R^{\prime 2} d r^{2}-R^{2} d \Omega^{2}
$$

and the evolution of the circumferential radius $R(t, r)$ and the density $\rho(r, t)$ is determined by the Einstein equations as

$$
\dot{R}^{2}=\frac{F(r)}{R}, \quad \rho=\frac{F^{\prime}}{R^{2} R^{\prime}} .
$$

In the above equations, a prime and a dot denote a derivative with respect to $r$ and $t$, respectively. Here $F(r)$ is the mass function which in case of the self-similar model is given by $F(r)=\lambda r$ when the scaling is $R=r$ at the singular epoch $t$ $=0$. Collapse is assumed to begin at some epoch $t<0$.

This self-similar model was discussed in Ref. [2]. The evolution of the circumferential radius is given by

$$
R^{3 / 2}=r^{3 / 2}\left(1-\frac{a t}{r}\right)
$$

where we have introduced $a=\frac{3}{2} \sqrt{\lambda}$. In this model, the initial density profile at $t=t_{i n}<0$ near the center is of the form

$$
\rho=\rho_{0}+\rho_{3} R^{3}+\rho_{6} R^{6}+\cdots .
$$

Here the coefficient $\rho_{3}=-(16 / 3) a^{-3}\left(-t_{i n}\right)^{5}$ is negative, in which case the central singularity is known to be globally naked for a range of values of $\lambda$. The values of higher order coefficients such as $\rho_{6}$ and $\rho_{9}$ are accordingly tuned so as to realize a self-similar solution.

The equation which determines the incoming (upper sign) and outgoing (lower sign) radial null geodesics on this background geometry is given by

$$
\mp \frac{d t}{d r}=R^{\prime}=\frac{1-\frac{1}{3} a t / r}{(1-a t / r)^{1 / 3}} .
$$

Following Ref. [2], we introduce the variable

$$
y=(1-a t / r)^{1 / 3} .
$$

Then the above equation is rewritten as

$$
\frac{d r}{r d y}=-\frac{9 y^{3}}{3 y^{4} \mp a y^{3}-3 y \mp 2 a}=-\sum_{i=1}^{4} \frac{3 A_{i}^{ \pm}}{\left(y-\alpha_{i}^{ \pm}\right)},
$$

where $\alpha_{i}^{ \pm}$are the roots of $3 y^{4} \mp a y^{3}-3 y \mp 2 a=0$, and the last equality defines the coefficients $A_{i}^{ \pm}$, which satisfy $\sum_{i=1}^{4} A_{i}^{ \pm}=1$. Equation (2.7) is integrated to obtain the solution

$$
\frac{r}{r_{0 \pm}}=\prod_{i=1}^{4}\left(y-\alpha_{i}^{ \pm}\right)^{-3 A_{i}^{ \pm}}
$$

Here $r_{0 \pm}$ is the integration constant which parametrizes different null rays. For the limit of small $r$ while keeping $t$ finite, $y$ goes to $\infty$. Then, in this limit, we find

$$
\frac{r}{r_{0 \pm}} \approx y^{-3}=\frac{1}{1-a t / r} \approx \frac{r}{a \xi}
$$

Here we have defined $\xi=-\left.t\right|_{r=0}$. From this relation, we can read that $r_{0 \pm}=a \xi$. We consider a pair of an outgoing radial null ray and an incoming one such that the latter is the reflection of the former at the center. Then both rays have the same value of $\xi$, and hence we conclude that

$$
r_{0+}=r_{0-}
$$

For a range of values of $\lambda$ the singularity forming in collapse is naked. In this case, the outgoing null ray emanating from the point at which the singularity first occurs, i.e., $r=0, t=0$, forms the Cauchy horizon. This null ray is given by $y=\alpha_{4}^{-}$where we assume $\alpha_{4}^{-}$is the largest real root among $\alpha_{i}^{-}$. Similarly, the incoming null ray terminating at the first singular point is also given by $y=\alpha_{4}^{+}$where, in the same way, $\alpha_{4}^{+}$is the largest real root among $\alpha_{i}^{+}$. Since we are considering the null rays close to the above limiting one, we expand the solution around $y=\alpha_{4}^{ \pm}$. Then we find

$$
\begin{aligned}
\frac{r}{a \xi} & \approx\left(y-\alpha_{4}^{ \pm}\right)^{-3 A_{4}^{ \pm}} \prod_{i=1}^{3}\left(\alpha_{4}^{ \pm}-\alpha_{i}^{ \pm}\right)^{-3 A_{i}^{ \pm}} \\
& \approx C_{ \pm}\left(t_{ \pm}(r)-t\right)^{-3 A_{4}^{ \pm}},
\end{aligned}
$$

where $t_{ \pm}(r)=(r / a)\left[1-\left(\alpha_{4}^{ \pm}\right)^{3}\right]$ and

$$
C_{ \pm}=\left[\frac{a}{3 r\left(\alpha_{4}^{ \pm}\right)^{2}}\right]^{-3 A_{4}} \prod_{i=1}^{3}\left(\alpha_{4}^{ \pm}-\alpha_{i}^{ \pm}\right)^{-3 A_{i}^{ \pm}} .
$$

As we have mentioned in the Introduction, we are considering a map between the sending time and the receiving time measured by an observer on a comoving shell. We denote the former time as $t_{1}$ and the latter time as $t_{2}$. Then, from Eqs. (2.10) and (2.11), we obtain

$$
\left(t_{-}\left(r_{s h}\right)-t_{2}\right)=\left[\frac{C_{-}}{C_{+}}\right]_{r=r_{s h}}^{1 /\left(3 A_{4}^{-}\right)}\left(t_{+}\left(r_{s h}\right)-t_{1}\right)^{A_{4}^{+} / A_{4}^{-}} \text {, }
$$

where $r_{s h}$ is the coordinate radius of the comoving shell on which the observer resides. Note that the result does not change even if we choose a very small value of $r_{s h}$. By a simple computation, we can show that $A_{4}^{+} / A_{4}^{-}$here is the quantity denoted by $\gamma$ in Ref. [2]. Hence, we find that the basic property of the map around the singularity is maintained in this new calculation, which does not use any information about the geometry away from the first singular point. 


\section{III. $\rho_{2}$ MODEL}

In the preceding section, we considered the self-similar model in which the second derivative of the density profile at the center vanishes, and the third derivative is in a certain negative range. In this section, we would like to consider more general cases in which the second derivative does not vanish.

What we would like to do here is to deduce an analogous map for the model with the $C^{\infty}$ initial density profile considered by Harada et al. $[3,4]$

$$
\rho(R)=\rho_{0}+\rho_{2} R^{2}+\cdots .
$$

We write the solution of Eq. (2.2) as

$$
R^{3}=\frac{9}{4} F(r)\left(t-t_{0}(r)\right)^{2},
$$

where $t_{0}(r)$ is an arbitrary function. By using the remaining gauge degrees of freedom we set

$$
t_{0}(r)=r \text {. }
$$

Then, the density profile near the center at some epoch $t$ $=t_{\text {in }}<0$ before hitting the singularity, which first appears at $t=0, r=0$, is given by

$$
\rho \approx \frac{4}{3 t_{\text {in }}^{2}}\left(1+\frac{2}{t_{\text {in }}}\left[r+\frac{F}{F^{\prime}}\right]\right) .
$$

For small $r$, we assume $F(r)=\alpha r^{\mu}+O\left(r^{\mu}\right)$. Then $R^{3} \propto r^{\mu}$ for small $r$ at $t=t_{i n}$. Requiring that the second term in the round brackets of Eq. (3.4), $r+F / F^{\prime}$, is proportional to $R^{2}$, we find that $\mu$ should be chosen as $3 / 2$. Then the condition $\rho_{2}<0$ is automatic, and the singularity at the center turns out to be at least locally naked.

Now let us consider a model truncated at the leading order for the expansion of $F(r)$, i.e., we suppose $F(r)=\alpha r^{3 / 2}$. Then the equation which determines the incoming and outgoing null geodesics is given by

$$
\frac{d t}{d r}=\mp \frac{7}{6} r^{1 / 6} \frac{1-\frac{3}{7} t / r}{(1-t / r)^{1 / 3}},
$$

where, for convenience, we have performed the following rescaling of variables,

$$
\begin{aligned}
& t \rightarrow\left(\frac{4}{9 \alpha}\right)^{2} t, \\
& r \rightarrow\left(\frac{4}{9 \alpha}\right)^{2} r .
\end{aligned}
$$

For small $r$, the right hand side of the above equation diverges, but this divergence is slower than $r^{-1}$. Hence the value of $t$ at $r=0$ dominates the solution for small $r$ unless it vanishes. Thus we consider an expansion of the solution in the form

$$
-t(r)=\xi-t_{1}(r)-t_{2}(r)-\cdots,
$$

where $\xi=-\left.t\right|_{r=0}$ as before.

To keep the notation simple, we introduce the function

$$
\mathcal{F}(s)=\frac{1-(3 s / 7)}{(1-s)^{1 / 3}} \text {. }
$$

Then, substituting the above ansatz (3.6) into Eq. (3.5), we get the equations which determine $t(r)$ order by order:

$$
\begin{aligned}
& \frac{d t_{1}}{d r}= \mp \frac{7}{6} r^{1 / 6} \mathcal{F}(-\xi / r), \\
& \frac{d t_{2}}{d r}=\mp \frac{7}{6} r^{1 / 6} \mathcal{F}^{\prime}(-\xi / r) \frac{t_{1}}{r}, \\
& \frac{d t_{3}}{d r}=\mp \frac{7}{6} r^{1 / 6}\left[\mathcal{F}^{\prime}(-\xi / r) \frac{t_{2}}{r}\right. \\
&\left.+\frac{1}{2} \mathcal{F}^{\prime \prime}(-\xi / r) \frac{t_{1}^{2}}{r^{2}}\right] .
\end{aligned}
$$

Recall that

$$
\frac{7}{6} r^{1 / 6} \mathcal{F}(-\xi / r)=\frac{d R(-\xi, r)}{d r}
$$

which relation is not at all accidental but follows from the starting expression for the geodesic equation $d t / d r=\mp R^{\prime}$. Using this relation, we can calculate $t_{1}(r)$ as

$$
t_{1}(r)=\mp R(-\xi, r)=\mp r^{7 / 6}(1+\xi / r)^{2 / 3} .
$$

The next order term is evaluated as

$$
\begin{aligned}
t_{2} & =\frac{7}{6} \int_{0}^{r} d r^{\prime} r^{\prime 1 / 3}\left(1+\xi / r^{\prime}\right)^{2 / 3} \mathcal{F}^{\prime}\left(-\xi / r^{\prime}\right) \\
& =\frac{7}{6} \xi^{8 / 6} \int_{0}^{r / \xi} d \mu \mu^{1 / 3}(1+1 / \mu)^{2 / 3} \mathcal{F}^{\prime}(-1 / \mu) .
\end{aligned}
$$

Expanding the integrand for large $r / \xi$, the asymptotic form of the integration is evaluated as

$$
\begin{aligned}
& \int_{0}^{r / \xi} d \mu \mu^{1 / 3}(1+1 / \mu)^{2 / 3} \mathcal{F}^{\prime}(-1 / \mu) \\
& \quad=C_{2}-\frac{(r / \xi)^{4 / 3}}{14}\left(1+\frac{28}{3}(\xi / r)+\cdots\right) .
\end{aligned}
$$

Here $C_{2}$ is the integration constant, which cannot be determined by integrating the expression expanded for large $r / \xi$. Except for the term containing $C_{2}$, all other terms are completely determined by the asymptotic expansion of the integrand. One may notice that we have not introduced the corresponding constant for $t_{1}(r)$. In the case of $t_{1}(r)$ we can see that this constant vanishes by looking at the explicit expression which is written in terms of $R$. It will be worth stressing that this cancellation of the integration constant is not due to the assumed form of $R$.

Also for $t_{3}(r)$, we can do a similar calculation as 


$$
\begin{aligned}
t_{3}= & \mp \frac{7}{6} \xi^{9 / 6} \int_{0}^{r / \xi} d \nu \nu^{1 / 6}\left\{\frac{7}{6 \nu} \mathcal{F}^{\prime}(-1 / \nu) \int_{0}^{\nu} d \mu \mu^{1 / 3}(1\right. \\
& \left.+1 / \mu)^{2 / 3} \mathcal{F}^{\prime}(-1 / \mu)+\frac{\nu^{1 / 3}}{2} \mathcal{F}^{\prime \prime}(-1 / \nu)(1+1 / \nu)^{4 / 3}\right\} \\
= & \mp \frac{7}{6} C_{3} \xi^{9 / 6} \pm \frac{7}{9} C_{2} \xi^{8 / 6} r^{1 / 6}(1+\cdots) \\
& \mp \frac{11 r^{9 / 6}}{162}(1+\cdots) .
\end{aligned}
$$

Besides the terms containing the integration constants $C_{k}$, terms in $t_{k}$ have the structure of $\xi^{m} r^{k / 6-m+1}(m \geqslant 0)$.

The region of validity of the above expansion will be determined by comparing the first and second term. Requiring that $\left|t_{1}\right| / \xi \ll 1$, we find that the condition for the validity of the above expansion is satisfied for $r \ll \xi^{6 / 7}$.

For sufficiently small $\xi$, within this region of validity, there is an overlapping region at which the condition $\xi / r$ $\ll 1$ also holds. When this condition $\xi / r \ll 1$ is satisfied, we can expand the geodesic equation as

$$
\frac{d t}{d r}=\mp \frac{7}{6} r^{1 / 6}\left(1-\frac{2 t}{21 r}+\frac{5 t^{2}}{63 r^{2}}+\cdots\right)
$$

Then, it can be integrated iteratively as

$$
\begin{aligned}
t-D_{\mp}= & r^{7 / 6} \\
& +\left( \pm \frac{2}{3} D_{\mp} r^{1 / 6}-\frac{1}{12} r^{8 / 6}\right) \\
& +\left( \pm \frac{1}{9} D_{\mp}^{2} r^{-5 / 6}+\frac{7}{9} D_{\mp} r^{2 / 6} \mp \frac{11}{162} r^{9 / 6}\right) \\
& +\cdots,
\end{aligned}
$$

where $D_{\mp}$ is the integration constant. Terms in the $(l+1)$-th line in the right-hand side have the structure of $D_{\mp}^{l-n} r^{(7(n+1) / 6)-l}(l \geqslant n \geqslant 0)$. Comparing them with the terms in $t_{k}$, we find the correspondence with $l=m+k-1, n=k$. Corresponding terms in two different expressions have the same coefficient. In other words, the first term in each line of the right hand side basically corresponds to $t_{1}(r)$, the second term to $t_{2}(r)$ and so on. The relation between $D_{\mp}$ and $\xi$ is found by comparing the coefficient of the $r$-independent term. Then we have

$$
\begin{aligned}
D_{\mp}+O\left(D_{\mp}^{2}\right)= & -\xi+\frac{7}{6} C_{2} \xi^{8 / 6} \mp \frac{7}{6} C_{3} \xi^{9 / 6}+\frac{7}{6} C_{4} \xi^{10 / 6} \\
& +O\left(\xi^{11 / 6}\right) .
\end{aligned}
$$

Eliminating $\xi$ from this equation, we obtain the relation between $D_{+}$and $D_{-}$,

$$
D_{-}=D_{+}+\frac{7}{3} C_{3}\left(-D_{+}\right)^{3 / 2}+O\left(D_{+}^{11 / 6}\right) \text {. }
$$

This mapping formula has the same structure of singularity as that obtained for the map between incoming and outgoing null coordinates by Harada, Iguchi and Nakao numerically.

\section{INTERPRETATION}

The map that we have obtained in this paper is the map between the ingoing and outgoing intersection points of null rays measured by the proper time of the comoving observer. This mapping is not exactly the same as the mapping of the incoming and outgoing null coordinates naturally defined for the observers at null infinities. However, as we mentioned in the Introduction, the singular behavior of these two mappings is expected to be common because there is no singular behavior in the null rays connecting the shell at a fixed $r$ and the shell at infinity.

Then, in order to investigate the structure of the singular mapping, is it possible to set the location of shell $r_{s h}$ arbitrarily small? The answer is basically yes. In the above derivation, the only constraint on $r_{s h}$ is $r_{s h} \gg \xi$, but this constraint does not prevent us from choosing $r_{s h}$ arbitrary small. Since we are interested in the limiting behavior when the time of reflection $t=-\xi$ comes very close to the singularity $t=0$, we can assume that $\xi$ is arbitrarily small. In the preceding section, we considered a matching of two different expressions for the null geodesics. One is valid for small $r$ and the other for large $r$. At this moment, one may wonder why it was necessary to consider this matching although we are allowed to choose $r_{s h}$ arbitrarily small. The reason is as follows. In the $\xi \rightarrow 0$ limit, the region of validity of the solution for small $r$ shrinks to zero. Hence, the solution for small $r$ cannot describe the limiting behavior for any value of $r_{s h}$.

Anyway, the basic feature of the map is derived for arbitrarily small $r_{s h}$. This fact proves that it is determined by the geometry just around the central singularity as expected. This is unlike the case of the black hole for which the map is not determined by the local geometry near the center. Instead it is determined by the relationship between the regular interior coordinates and the external coordinates, near the event horizon.

Here we have considered a specific model for the initial density profile. However, we can expect that the basic structure of the map will be independent of the details of the model because it is determined by the geometry just near the point at which the singularity is first formed. In this small region, we would be able to neglect the higher order terms in the expansion of the density profile, although further consideration is necessary to give a rigorous proof for the statement that the structure of singularity in the mapping formula is totally determined by the leading term in the expansion of the initial density profile for general spherical dust collapse.

In this paper we have discussed models of spherically symmetric dust collapse. However, it would be of interest to generalize the prescription described in this paper to other matter models and to non-spherical collapse.

\section{ACKNOWLEDGMENTS}

It is a pleasure to thank T. Harada, H. Iguchi, A. Ishibashi, H. Kodama, and K. Nakao for useful discussions. T.T. acknowledges support from Monbusho Grant-in-Aid No. 1270154. T.P.S. acknowledges the partial support of the Fundação para a Ciência e a Tecnologia (FCT), Portugal under contract number SAPIENS/32694/99. 
[1] L. H. Ford and L. Parker, Phys. Rev. D 17, 1485 (1978).

[2] S. Barve, T. P. Singh, C. Vaz, and L. Witten, Nucl. Phys. B532, 361 (1998).

[3] T. Harada, H. Iguchi, and K. Nakao, Phys. Rev. D 61, 101502 (2000).

[4] T. Harada, H. Iguchi, and K. Nakao, Phys. Rev. D 62, 084037 (2000).

[5] T. P. Singh and Cenalo Vaz, Phys. Lett. B 481, 74 (2000).
[6] W. A. Hiscock, L. G. Williams, and D. M. Eardley, Phys. Rev. D 26, 751 (1982).

[7] S. Barve, T. P. Singh, Cenalo Vaz, and Louis Witten, Phys. Rev. D 58, 104018 (1998).

[8] S. Barve, T. P. Singh, and C. Vaz, Phys. Rev. D 62, 084021 (2000).

[9] T. Harada, H. Iguchi, K. Nakao, T. P. Singh, T. Tanaka, and C. Vaz, gr-qc/0010101. 